\title{
MAPPING THE CLUMPY STRUCTURES WITHIN SUBMILLIMETER GALAXIES USING LASER-GUIDE STAR ADAPTIVE OPTICS SPECTROSCOPY
}

\author{
Karín Menéndez-Delmestre ${ }^{1,2,3,9}$, Andrew W. Blain ${ }^{2,4}$, Mark SwinbanK ${ }^{5}$, Ian Smail ${ }^{5}$, \\ Rob J. Ivison ${ }^{6,7}$, Scott C. Chapman ${ }^{8}$, and Thiago S. Gonçalves ${ }^{1}$ \\ ${ }^{1}$ Observatório do Valongo, Universidade Federal do Rio de Janeiro, Ladeira do Pedro Antônio 43, Saúde, Rio de Janeiro, \\ RJ 20080-090, Brazil; kmd@ astro.ufrj.br \\ ${ }^{2}$ California Institute of Technology, MC 105-24, Pasadena, CA 91125, USA \\ 3 The Observatories of the Carnegie Institution for Science, 813 Santa Barbara St., Pasadena, CA 91101, USA \\ ${ }^{4}$ University of Leicester, University Road, Leicester LE1 7RH, UK \\ ${ }^{5}$ Institute for Computational Cosmology, Durham University, Durham DH1 3LE, UK \\ ${ }^{6}$ UK Astronomy Technology Centre, Blackford Hill, Edinburgh EH9 3HJ, UK \\ ${ }^{7}$ Institute for Astronomy, Blackford Hill, Edinburgh EH9 3HJ, UK \\ ${ }^{8}$ Institute of Astronomy, Madingley Road, Cambridge CB3 OHA, UK \\ Received 2010 August 1; accepted 2013 February 4; published 2013 April 8
}

\begin{abstract}
We present the first integral-field spectroscopic observations of high-redshift submillimeter-selected galaxies (SMGs) using Laser-Guide Star Adaptive Optics. We target $\mathrm{H} \alpha$ emission of three SMGs at redshifts $z \sim 1.4-2.4$ with the OH-Suppressing Infrared Imaging Spectrograph on Keck. The spatially resolved spectroscopy of these galaxies reveals unresolved broad-H $\alpha$ line regions (FWHM $>1000 \mathrm{~km} \mathrm{~s}^{-1}$ ) likely associated with an active galactic nucleus (AGN) and regions of diffuse star formation traced by narrow-line $\mathrm{H} \alpha$ emission (FWHM $\lesssim 500 \mathrm{~km} \mathrm{~s}^{-1}$ ) dominated by multiple $\mathrm{H} \alpha$-bright stellar clumps, each contributing $1 \%-30 \%$ of the total clump-integrated $\mathrm{H} \alpha$ emission. We find that these SMGs host high star formation rate surface densities, similar to local extreme sources, such as circumnuclear starbursts and luminous infrared galaxies. However, in contrast to these local environments, SMGs appear to be undergoing such intense activity on significantly larger spatial scales as revealed by extended $\mathrm{H} \alpha$ emission over 4-16 kpc. $\mathrm{H} \alpha$ kinematics show no evidence of ordered global motion as would be found in a disk, but rather large velocity offsets $\left(\sim\right.$ few $\left.\times 100 \mathrm{~km} \mathrm{~s}^{-1}\right)$ between the distinct stellar clumps. Together with the asymmetric distribution of the stellar clumps around the AGN in these objects, it is unlikely that we are unveiling a clumpy disk structure as has been suggested in other high-redshift populations of star-forming galaxies. The SMG clumps in this sample may correspond to remnants of originally independent gas-rich systems that are in the process of merging, hence triggering the ultraluminous SMG phase.
\end{abstract}

Key words: galaxies: high-redshift - galaxies: nuclei - galaxies: starburst - galaxy: kinematics and dynamics submillimeter: galaxies - techniques: imaging spectroscopy

Online-only material: color figures

\section{INTRODUCTION}

The details of stellar mass assembly are crucial for a full understanding of galaxy evolution. Most of the present-day stellar mass in massive galaxies was already in place at $z \sim$ 1 , and the bulk of stellar build-up apparently took place at $1 \lesssim z \lesssim 3$ (e.g., Dickinson et al. 2003; Pérez-González et al. 2008). A population of starbursting galaxies at $z \sim 2$ that have been popular candidates to be the progenitors of the most massive galaxies present at $z \sim 0$ are the so-called submillimeter galaxies (SMGs; Blain et al. 2002) identified by submillimeter and millimeter surveys at $\lambda \sim 850-125 \mu \mathrm{m}$ (Smail et al. 1997; Hughes et al. 1998; Barger et al. 1998, 1999; Eales et al. 1999; Bertoldi et al. 2000; Cowie et al. 2002; Scott et al. 2002; Borys et al. 2003; Webb et al. 2003b; Coppin et al. 2005; Younger et al. 2007; Weiß et al. 2009; Wardlow et al. 2011). With large infrared luminosities $\left(L_{8-1000 \mu \mathrm{m}} \gtrsim 10^{12} L_{\odot}\right)$ that translate into star formation rates (SFRs) of SFRs $\gtrsim 100-1000 M_{\odot} \mathrm{yr}^{-1}$ (Kennicutt 1998), SMGs could build the stellar bulk of a massive galaxy in under a few hundred million years (Lilly et al. 1999; Genzel et al. 2003).

Deep long-slit spectroscopic $\mathrm{H} \alpha$ observations of a large sample (>30) of SMGs with the Keck instrument NIRSPEC

\footnotetext{
9 NSF Astronomy and Astrophysics Postdoctoral Fellowship.
}

have provided crucial constraints on SFRs and dynamical masses of this population (SFR $\sim 1000 M_{\odot} \mathrm{yr}^{-1}, M_{\text {dyn }} \sim$ 1-2 $\times 10^{11} M_{\odot}$; Swinbank et al. 2004). These studies reported the presence of broad Balmer line emission $\left(\mathrm{FWHM}_{\mathrm{H} \alpha} \gtrsim\right.$ $1000 \mathrm{~km} \mathrm{~s}^{-1}$ ) in a large fraction of SMGs ( $\left.\gtrsim 40 \%\right)$, suggesting a direct view to the randomly moving high-velocity gas within the broad-line region of a central active galactic nucleus (AGN). Parallel studies at other wavelengths have revealed a mixture in the astrophysical nature of the underlying power sources in SMGs. Exploiting deep Chandra X-ray observations, Laird et al. (2010) show that 20\%-30\% SMGs host an AGN. However, AGN activity has been shown not to dominate the SMG bolometric luminosities (Alexander et al. 2005, 2008, based on X-ray observations; Pope et al. 2008; Menéndez-Delmestre et al. 2009, based on the mid-IR). High-resolution very long baseline interferometry radio studies of SMGs have also given us insight to the underlying AGN activity in SMGs (Biggs et al. 2010).

When both AGN and star formation activity co-exist, long-slit spectroscopic techniques face difficulties in disentangling their independent contributions. Estimates of SFR and dynamical mass based on $\mathrm{H} \alpha$ line information in SMGs thus retain the substantial caveat that in the presence of an AGN, the blended nuclear emission may result in the broadening and brightening of the $\mathrm{H} \alpha$ emission, potentially leading to AGN-contaminated 
measurements. Without spatially resolved information, it is difficult to disentangle the AGN contribution from the nebular emission.

Integral-field spectroscopy opens the possibility of investigating the properties of nebular emission at different scales, providing the spatially resolved information to allow a distinction in spectral properties across a galaxy. Furthermore, considering that dynamical information has proved difficult to extract from long-slit data (Swinbank et al. 2004), even for the less extreme and likely more ordered cases of optically selected Lyman-break galaxies (LBGs) at similar redshifts (Erb et al. 2003), an integral spectroscopic insight to the line emission is also the only viable probe of the distribution and dynamics of the gas in the inner galaxy.

Results from the SPectrometer for Infrared Faint Field Imaging on the ESO Very Large Telescope (VLT) first showed how two-dimensional (2D) marginally resolved seeing-limited spectroscopy could already reveal structure in line emission across an SMG at $z \simeq 2.5$, SMM J14011+0252 (Tecza et al. 2004). Thereafter, a number of studies using integral-field spectrographs at the VLT, Gemini, and UKIRT telescopes have explored the spatial distribution and velocity fields of $\mathrm{H} \alpha$ emission in a handful of SMGs (Swinbank et al. 2005, 2006; Nesvadba et al. 2007; Alaghband-Zadeh et al. 2012; Harrison 2012). Furthermore, high-resolution Hubble Space Telescope (HST) NICMOS/Advanced Camera for Surveys (ACS) imaging of 25 SMGs has revealed a mix of morphologies from compact, single component to clumpy extended structures (Swinbank et al. 2010), either due to structured dust extinction on a smooth disk distribution or star formation activity concentrated in clumps. Evidence for clumpy star formation is not limited to SMGs but has also been observed in the rest-frame $\mathrm{UV} /$ optical continuum of less extreme star-forming galaxies at intermediate and high redshifts (e.g., Conselice et al. 2004; Elmegreen et al. 2004a, 2004b; Elmegreen \& Elmegreen 2005; Law et al. 2007a; Overzier et al. 2010; Förster Schreiber et al. 2011). Spatially resolved observations of the ionized gas have provided insight to the underlying kinematics to help discern between a clumpy-disk and a merger scenario (e.g., Keck/OSIRIS: Stark et al. 2008; Basu-Zych et al. 2009; Melbourne et al. 2009; Wright et al. 2009; Law et al. 2009; Gonçalves et al. 2010; Jones et al. 2010; Wisnioski et al. 2012; VLT/SINFONI: Förster Schreiber et al. 2009; Genzel et al. 2011).

Recent integral-field unit (IFU) work by Alaghband-Zadeh et al. (2012) with the GEMINI-NIFS and VLT-SINFONI instruments reveals the predominance of clumpy structure in a sample of nine SMGs at $2.0<z<2.7$ characterized by multiple galactic-scale subcomponents with an average projected separation of $\sim 8 \mathrm{kpc}$ and velocity offsets of $\sim 200 \pm 100 \mathrm{~km} \mathrm{~s}^{-1}$ reminiscent of merging systems. Even higher internal velocities up to $\sim 800 \mathrm{~km} \mathrm{~s}^{-1}$ are identified by Harrison (2012) in a sample of eight SMGs at $1.4<z<3.4$, but these are based on [O III] line emission and attributed to large-scale AGN outflows.

A theoretical framework for the formation of clumpy structures at high redshifts is actively being developed in recent years, with merger (e.g., Hayward et al. 2011) and disk fragmentation simulations successfully reproducing the observational evidence (e.g., Immeli et al. 2004; Dekel et al. 2009; Bournaud et al. 2009, 2011). Within the latter scenario, disk fragmentation into giant clumps results from gravitational instabilities triggered by disk-wide turbulence due to rapid gas cooling. The otherwise smooth rotation pattern expected for rotating disks is highly distorted by the velocity offsets of the bright clumps (Immeli et al. 2004). This "clumpy stage" is a short-lived phase in the evolutionary scenario of disk galaxies, where disk fragmentation is rapidly followed by clump migration to the center of the galaxy building the central bulge in only a few $\sim 10^{8}$ yr. Dekel et al. (2009) propose a slightly different scenario where clumpy disks persist for a longer term and are thus more prevalent in the observed population of high-redshift galaxies. Within this scenario, cold flows provide a continuous and rapid stream of smooth and clumpy gas along galactic-scale filaments that feed galaxy star formation, keeping the disk gravitationally unstable without destroying it.

In this paper, we present the first integral-field spectroscopic observations of SMGs aided with Laser-Guide Star Adaptive Optics (LGS-AO), unveiling spatially resolved details on the

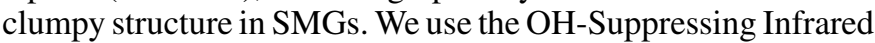
Imaging Spectrograph (OSIRIS; Larkin \& Versteegh 2006) on the Keck II telescope to study the $\mathrm{H} \alpha$ emission in three SMGs in the redshift range $z \sim 1.4-2.4$. The superior spatial resolution provided by these observations compared to previous work allows us for the first time to investigate down to kpc-scale detail the internal kinematics and the distribution of spectral properties in these galaxies in the rest-frame optical. We describe our sample selection, observing strategy, and the steps comprising the reduction and analysis of the science spectra in Section 2. Our results are presented in Section 3 and discussed in Section 4. We give our conclusions in Section 5. We assume a $\Lambda \mathrm{CDM}$ cosmology, with $H_{0}=71 \mathrm{~km} \mathrm{~s}^{-1} \mathrm{Mpc}^{-1}, \Omega_{M}=0.27$, and $\Omega_{\Lambda}=0.73$.

\section{SAMPLE SELECTION, OBSERVATIONS, AND ANALYSIS}

OSIRIS, with spectral resolution $R \simeq 3000-3800$, is a lenslet-based spectrograph that allows for a spatial sampling ranging from $0^{\prime} .02$ to $0^{\prime} .1$, depending on the selected lenslet scale. It is designed to be used with the Keck LGS-AO system (Wizinowich et al. 2006; van Dam et al. 2006). LGS-AO allows for atmospheric distortion corrections to be derived from parallel observing of a spatially coincident laser excited sodium beacon in the upper atmosphere and thus enables close to diffraction-limited resolution in ground-based observations. At the typical redshifts of SMGs, $z \sim 2$ (Chapman et al. 2005), this corresponds to kpc-scale spatial resolution.

Our targets are from the radio-identified sample of SMGs in Chapman et al. (2005). We selected our science targets carefully to optimize OSIRIS observations, including $\mathrm{H} \alpha$ signal-to-noise ratio $(\mathrm{S} / \mathrm{N})$, low sky-line contamination, and performance of AO correction. We took advantage of existing near-IR longslit $\mathrm{H} \alpha$ spectroscopy to select SMGs with the brightest $\mathrm{H} \alpha$ lines, $S_{\mathrm{H} \alpha} \gtrsim 10^{-15} \mathrm{erg} \mathrm{s}^{-1} \mathrm{~cm}^{-2}$ (Swinbank et al. 2004; Takata et al. 2006), to maximize detection $\mathrm{S} / \mathrm{N}$ with OSIRIS. With redshifts in hand we selected SMGs for which the redshifted $\mathrm{H} \alpha$ emission does not fall on bright $\mathrm{OH}$ sky emission lines, in order to minimize sky contamination. Since the LGS-AO system requires the presence of a relatively bright $(R \lesssim 18)$ star within $1^{\prime}$ of the science target in order to derive AO-corrections, we selected SMGs with such nearby tip-tilt stars (hereafter TTstar). The three SMG targets comprising our sample are listed in Table 1.

The submillimeter fluxes of the SMGs in our sample $\left(S_{850 \mu \mathrm{m}} \sim 8 \mathrm{mJy}\right.$; Chapman et al. 2005), together with their stellar masses $\left(M_{\star} \sim 7 \times 10^{10} M_{\odot}\right.$; Hainline et al. 2011), set them well within the range of typical values for the SMG population as a whole. However, we note that by selecting galaxies that 
Table 1

Summary of OSIRIS Observations

\begin{tabular}{lccc}
\hline \hline SMM J & 030227.73 & 123549.44 & 163650.43 \\
\hline R.A. $^{\mathrm{a}}$ & $03: 02: 27.73$ & $12: 35: 49.44$ & $16: 36: 50.43$ \\
Decl. $^{\mathrm{a}}$ & $+00: 06: 53.5$ & $+62: 15: 36.8$ & $+40: 57: 34.5$ \\
$z_{\mathrm{H}_{\alpha}}{ }^{\mathrm{b}}$ & 1.4076 & 2.2032 & 2.3850 \\
Filter $^{\text {Wavelength range }}{ }^{\mathrm{c}}(\mathrm{nm})$ & $1532.0-1609.5$ & $2036.2-2140.7$ & $2121.3-2229.4$ \\
Wlate scale & $0^{\prime \prime} 1$ & $0 .{ }^{\prime} .05$ & 0. \\
Exposure & $12.6 \mathrm{ks}$ & $10.8 \mathrm{ks}$ & $12.6 \mathrm{ks}$ \\
\hline
\end{tabular}

Notes.

${ }^{a}$ Radio center from Chapman et al. (2005).

b From Swinbank et al. (2004).

${ }^{c}$ Cut-on and cutoff wavelengths.

are particularly bright in $\mathrm{H} \alpha$ compared to the rest of the SMG population, we introduce an important bias favoring SMGs with strong emission lines and likely hosting AGNs. Incidentally, all three SMGs in our sample display AGN signatures either in the near-IR, the mid-IR, or X-rays. We discuss these individually in Section 3.1.

Our OSIRIS observations include a combination of the 0.05 and the 0 .'1 lenslet scales, which provide the largest fields of view (FOVs: $\sim 2$ 2. $4 \times 3$ 3.2 and $\sim 4$ ". $8 \times 6$ 6."4, respectively) and also have the advantage of allowing us to maximize the $\mathrm{S} / \mathrm{N}$ per lenslet, crucial for the study of faint sources. The science integrations comprise sets of standard ABBA sequences, each comprising four 15-minute frames taken at two positions separated by 0 .'5 to allow for on-object dithering. We followed each of these sets with a dedicated-sky integration frame, offset by $15^{\prime \prime}$ from the science target. In Table 1 , we provide details of the observing setup for each of our targets.

To process our data, we used the OSIRIS Data Reduction System pipeline ${ }^{10}$ which comprises a collection of individual IDL modules, each performing a specific task to ultimately extract each lenslet's spectrum from the raw 2D data frame and spatially reconstruct the image using a reference map-a rectification matrix - where each pixel within the detector is assigned back to its corresponding lenslet position. The result is a three-dimensional data cube with two spatial axes $(x, y)$ and a third axis for wavelength $(\lambda)$, calibrated according to the corresponding rectification matrix available for the particular filter and lenslet scale.

We improve the sky subtraction by subtracting a supersky 2D image from our science cubes. The supersky image results from median-combining the pipeline-processed sky cubes and collapsing these along the $\lambda$-range around the redshifted $\mathrm{H} \alpha$ wavelength ( $\Delta \lambda \simeq \lambda_{\mathrm{H} \alpha} \pm 600 \AA$ ). We also subtract the median flux level within each wavelength channel in the science cubes to eliminate the residual background plateau. Finally, we applied the sky-subtraction routine developed by Davies (2007), specifically tailored for near-IR integral-field spectra, taking into account the temporal variations in absolute fluxes of $\mathrm{OH}$ lines as well as in their individual fluxes relative to each other. This final step contributed a modest increase in $\mathrm{S} / \mathrm{N}(\lesssim 1.5)$ in our science images.

\subsection{Ho Maps}

We construct $\mathrm{H} \alpha$ intensity, velocity $\left(v_{\mathrm{H} \alpha}\right)$, and line-width $\left(\sigma_{\mathrm{H} \alpha}\right)$ maps for each target to help us understand the distribution of $\mathrm{H} \alpha$ properties across the SMGs in our sample (see

\footnotetext{
10 http://www2.keck.hawaii.edu/inst/osiris/tools/
}

Figures 1-6). Following a similar approach to Law et al. (2009) and Gonçalves et al. (2010), we convolve the individual image frames at each wavelength channel with a 2D Gaussian kernel ( $F W H M=1.5$ pixels) in an effort to improve the $\mathrm{S} / \mathrm{N}$ while remaining below the spatial resolution limit of our observations as determined by our point-spread function (PSF; FWHM 0'.10-0'.25), measured from the TT-star peak-up imaging. For SMM J123549.44 and SMM J163650.43, we also smooth the one-dimensional (1D) spectra at each spatial pixel with a $2 \AA$ and $5 \AA$ wide Gaussian kernel, respectively, to better distinguish the line emission from the underlying noise.

Imposing a set of line-search thresholds for the $\mathrm{H} \alpha$ line width $\left(\lesssim 2500 \mathrm{~km} \mathrm{~s}^{-1}\right), \mathrm{S} / \mathrm{N}$ with respect to an off-source region ( $\gtrsim$ ), and velocity offset relative to the long-slit $\mathrm{H} \alpha$ redshift $\left(\lesssim 1000 \mathrm{~km} \mathrm{~s}^{-1}\right)$ for our targets from Swinbank et al. (2004), we fit single Gaussian profiles to the $\mathrm{H} \alpha$ line emission along the dispersion direction at each spatial pixel. We use the collection of these Gaussian fits, in particular the central wavelength and the line width (corrected for instrumental width) at each spatial pixel, to construct an $\mathrm{H} \alpha$ velocity map, reflecting the relative spectral position of the line centroids, and an $\mathrm{H} \alpha$ linewidth map characterizing the velocity dispersions of the $\mathrm{H} \alpha$ emission. Note that only the Gaussian fits complying with our imposed thresholds are used for the velocity and line-width maps, while other ones are disregarded (i.e., empty pixel in the resulting maps). The selection of these $\mathrm{S} / \mathrm{N}$, velocity, and line-width thresholds were not established a priori, but tuned carefully to each object, in order to eliminate spurious elements from the resulting maps. We produce $\mathrm{H} \alpha$ intensity maps by collapsing the final science data cube along the dispersion direction, stacking the science data cube's flux over a range $\Delta v \simeq 1000-2000 \mathrm{~km} \mathrm{~s}^{-1}$ centered at the redshifted $\mathrm{H} \alpha$ line. In Figures 2, 4, and 6, we show $\mathrm{H} \alpha$ velocity and line-width maps with overlaid $\mathrm{H} \alpha$ intensity contours for SMM J030227.73, SMM J123549.44, and SMM J163650.43, respectively. We oversample these maps by a factor of two to improve their visual appearance. We also show the 1D spectra integrated over several kiloparsec-sized $\mathrm{H} \alpha$-bright regions, which are labeled in the maps shown in Figures 1, 3, and 5.

We flux calibrate the $\mathrm{H} \alpha$ line emission in our OSIRIS observations using as a reference the $\mathrm{H} \alpha$ line fluxes from longslit spectroscopy by Swinbank et al. (2004), where slit loss correction has been performed based on $K$-band images. We set the galaxy-integrated long-slit $\mathrm{H} \alpha$ fluxes equal to the integrated OSIRIS flux resulting from collapse of the science cube along the dispersion direction $\left(\Delta v \simeq 1000-2000 \mathrm{~km} \mathrm{~s}^{-1}\right)$ centered at the redshifted $\mathrm{H} \alpha$ line. By adopting this approach, we ensure that we are not artificially boosting the $\mathrm{H} \alpha$ emission within the brightest clump regions. In principle, peak-up imaging of the TT-star for each science target is also a viable reference to perform absolute flux calibrations. However, we note that when using the AO technique, a large flux uncertainty arises from the imperfect channeling of light into the AO-corrected PSF. Following detailed modeling of this issue by Law et al. (2007b), the total flux uncertainty for the reference TT-star flux corresponds to at least $\sim 30 \%$. Furthermore, because flux calibration requires also taking into account the spatial offset between the science target and the TT-star to consider the true PSF at the science target location, the uncertainties in absolute flux calibration based on TT-star observations increase significantly.

In order to disentangle the diffuse $\mathrm{H} \alpha$ component $\left(\sim 0.5-2^{\prime \prime}\right)$ from the compact one (FWHM $\left.\sim 0^{\prime \prime} .2-0^{\prime \prime} 4\right)$, we rely on a 

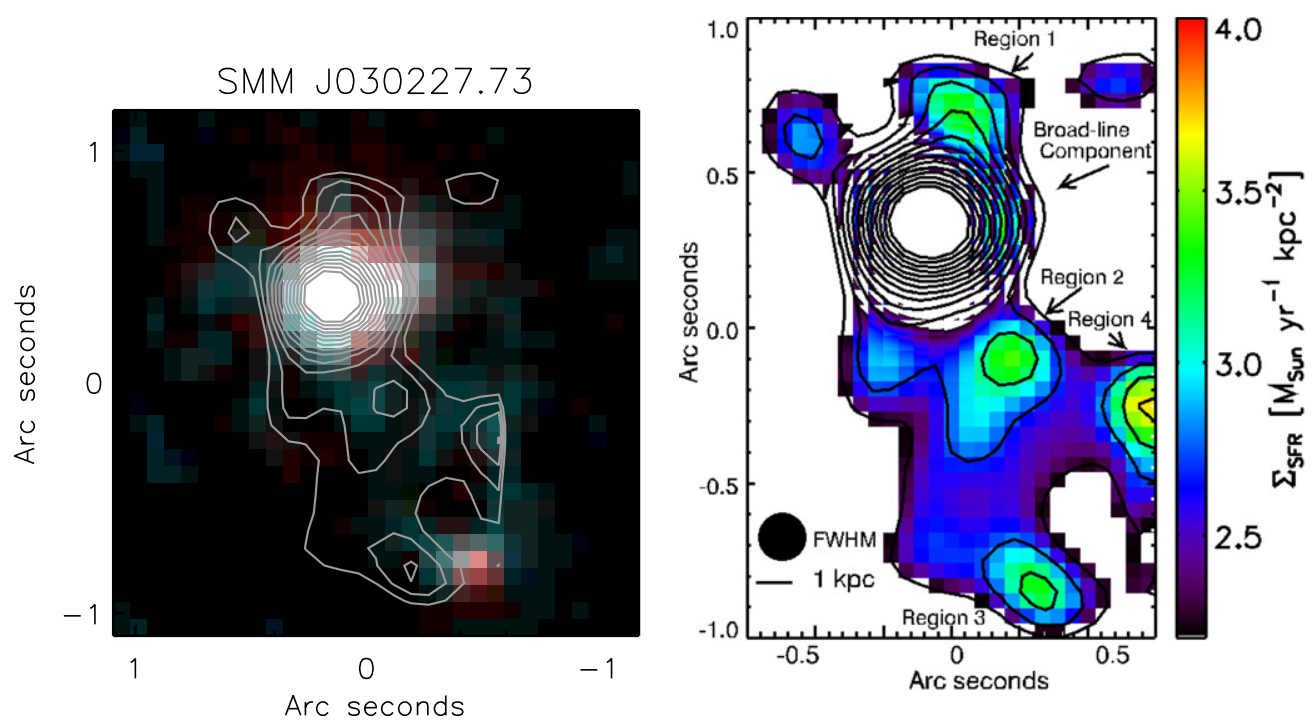

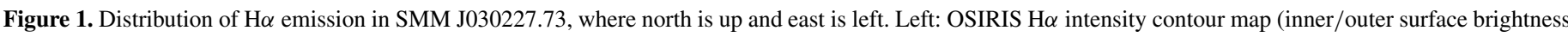

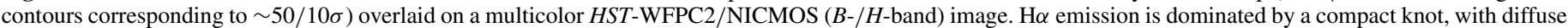

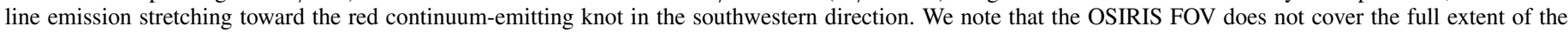

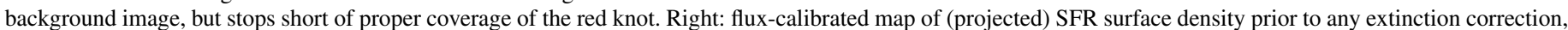

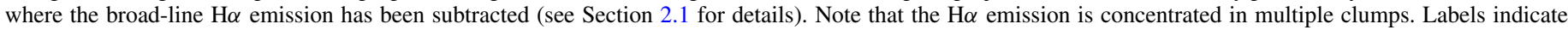

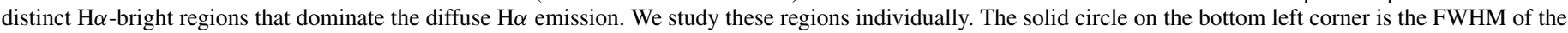
TT-star taken just prior to the science exposures to represent the PSF of the observations; the horizontal bar represents 1 kpc at the redshift of the target.

(A color version of this figure is available in the online journal.)

target-specific PSF determined from peak-up imaging of the TT-star to subtract the compact region of high-S/N line emission from each map. This is particularly important considering the potential spread of emission beyond the AO-corrected PSF that may escape the immediate regions of these high-S/N regions of emission. In this way, we avoid potential contamination and ensure the best separation of the diffuse and compact $\mathrm{H} \alpha$ emission components. The PSF-subtracted images that we use to investigate diffuse emission are shown in Figures 1, 3, and 5 for SMM J030227.73, SMM J123549.44, and SMM J163650.43, respectively.

\section{RESULTS}

The OSIRIS $\mathrm{H} \alpha$ intensity maps are shown in Figures 1, 3, and 5 as contours overlaid on near-IR images tracing the restframe optical continuum of these galaxies. The $\mathrm{H} \alpha$ contours allow us to map the overall spatial distribution of the line emission and to distinguish between unresolved kiloparsecsized compact regions of high-S/N emission and a more diffuse component, where the former are likely associated with nuclear activity, either an AGN or a compact starburst, and the latter corresponds to star formation. Note the prevalence of clumpy structure in the sources on 1-2 kpc scales. The line-width and velocity maps for each of our targets in Figures 2, 4, and 6 allow us to probe for velocity offsets between distinct components in each system and for the presence of AGNs, as revealed by large line widths (FWHM $>1000 \mathrm{~km} \mathrm{~s}^{-1}$ ). From these OSIRIS observations, we derive clump-integrated velocity dispersions $\left(\sigma_{\mathrm{H} \alpha}\right)$, SFRs, and SFR surface densities $\left(\Sigma_{\mathrm{SFR}}\right)$. We list these in Table 2.

\subsection{Notes on Individual SMGs}

\subsubsection{SMM J030227.73}

SMM J030227.73 (aka CUDSS 3.15) was first detected and identified by Webb et al. (2003a). Optical and near-IR spec- tra by Chapman et al. (2005) and Swinbank et al. (2004) place this galaxy at $z=1.408$, with a high $[\mathrm{N}$ II $] / \mathrm{H} \alpha$ ratio $([\mathrm{N} \mathrm{II}] / \mathrm{H} \alpha=1.38 \pm 0.07$; Swinbank et al. 2004) suggesting the presence of AGN activity. Harrison (2012) also identify spatially unresolved $(\leqslant 3.6 \mathrm{kpc})$, broad emission $(\mathrm{FWHM}=$ $900 \pm 300 \mathrm{~km} \mathrm{~s}^{-1}$ ) in galaxy-integrated [O III] emission for this object. Furthermore, Spitzer mid-IR spectroscopic follow-up of this object shows a substantial excess in mid-IR continuum, suggesting a significant AGN contribution (MenéndezDelmestre et al. 2009; see also Hainline et al. 2009). The nearIR continuum map of SMM J030227.73 in Figure 1 shows that continuum emission is dominated by a bright, compact source, with a much fainter secondary component $\sim 1^{\prime \prime} .3(\sim 11 \mathrm{kpc})$ to the southwest. With a high $[\mathrm{N}$ II $] / \mathrm{H} \alpha$ ratio, this secondary component has been tentatively identified with an AGN by Swinbank et al. (2006). A faint $\mathrm{H} \alpha$-bright bridge was also detected between the two point-source components in the non-AO $\mathrm{H} \alpha$ observations of Swinbank et al. (2006).

The OSIRIS H $\alpha$ contours in Figure 1 show a compact peak of $\mathrm{H} \alpha$ emission (FWHM $\simeq 0$ '.36, corresponding to $\sim 3 \mathrm{kpc}$ ) that coincides with the continuum-bright compact region. Although the southwest secondary continuum-detected compact source lies just outside our FOV, OSIRIS observations allow us to spatially resolve the bridge region between the two continuumdetected compact components. As shown in Figure 1, $\mathrm{H} \alpha$ contours highlight the bridge region of diffuse $\mathrm{H} \alpha$ emission extending out to $\gtrsim 1^{\prime \prime}(8.5 \mathrm{kpc})$ to the southwest of the central bright source. In Figure 2, we show the $\mathrm{H} \alpha$ line-width $\left(\sigma_{\mathrm{H} \alpha}\right)$ and velocity maps for SMM J030227.73 with overlaid $\mathrm{H} \alpha$ flux contours. We also show the 1D $\mathrm{H} \alpha$ spectra extracted from distinct spatial regions: the bright point source and four distinct clumps within the diffuse $\mathrm{H} \alpha$ emission, three of which lie in the $\mathrm{H} \alpha$-bright bridge region extending to the southwest. The bright, compact knot from the central source stands out at an $\mathrm{S} / \mathrm{N} \sim$ 40 with broad-H $\alpha$ emission $\left(\sigma_{\text {rest }} \gtrsim 1000 \mathrm{~km} \mathrm{~s}^{-1}\right.$; see 1D spectrum in Figure 2), while to the southwest fainter and diffuse 

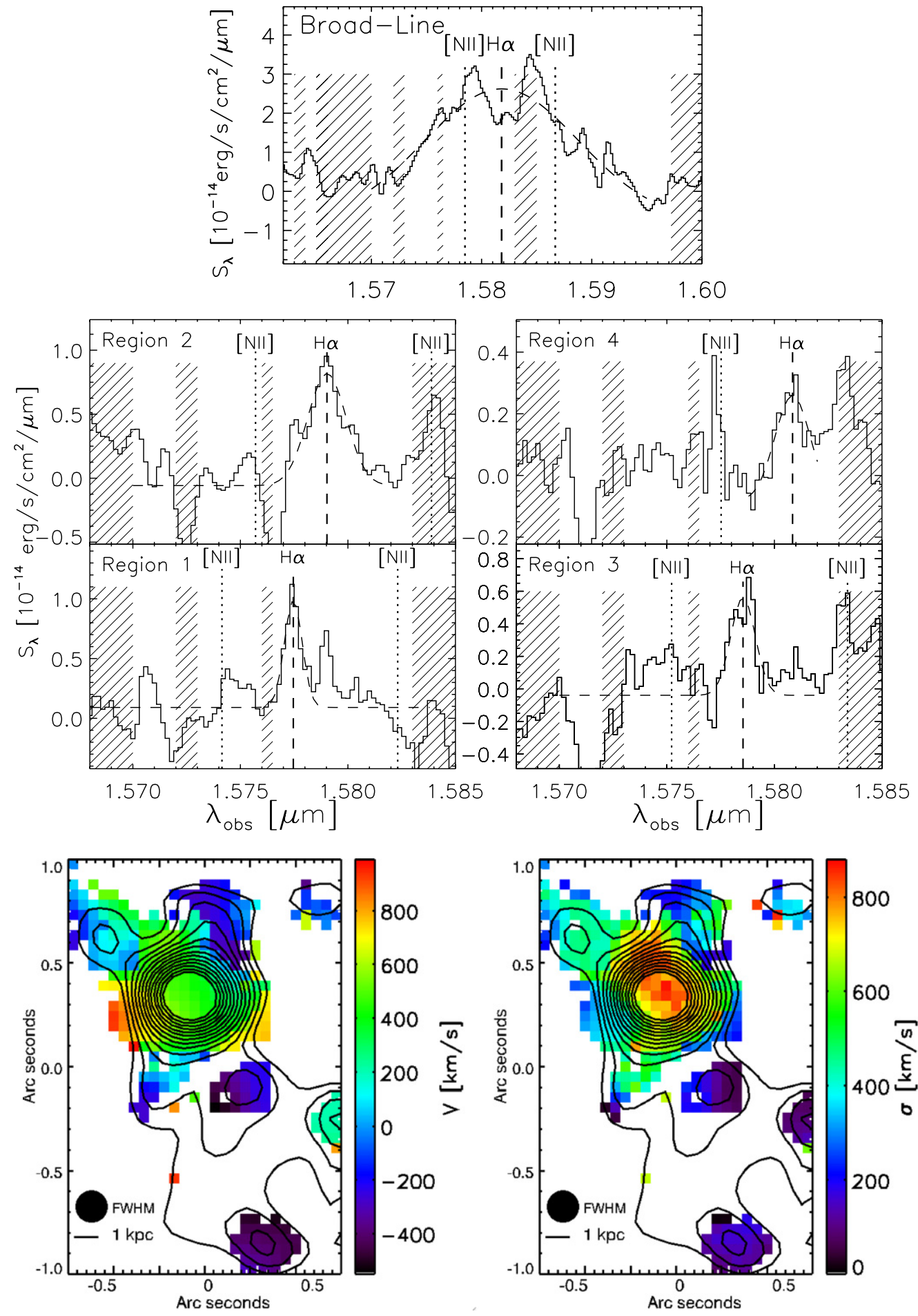

Figure 2. Spectral properties and kinematic maps for SMM J030227.73. Top: $\mathrm{H} \alpha$ 1D spectra extracted from individual $\mathrm{H} \alpha$-bright regions (see Figure 1): the broad-H $\alpha$ component corresponds to the dominating point source and the four narrow-H $\alpha$ components are associated to distinct clumps, one adjacent to the broad-line region and three within the diffuse region stretching in the southwest direction. The location of bright $\mathrm{OH}$ lines are shown by diagonally hashed vertical columns. Bottom left: $\mathrm{H} \alpha$ velocity map revealing no global velocity gradient across the galaxy, but velocity offsets $\left(\sim 200-600 \mathrm{~km} \mathrm{~s}^{-1}\right)$ between the bright point-source component and the other $\mathrm{H} \alpha$-bright clumps. Bottom right: $\mathrm{H} \alpha$ line-width $\left(\sigma_{\mathrm{H} \alpha}\right)$ map showing the point-source component with broad-H $\alpha$ emission relative to that of the remaining clumps. We attribute the broad-H $\alpha$ emission to AGN activity, while the emission within the remaining clumps and diffuse region is more likely associated with star formation.

(A color version of this figure is available in the online journal.)

narrow-H $\alpha$ emission appears at a lower, but still significant $\mathrm{S} / \mathrm{N} \sim 10-20\left(\sigma_{\text {rest }} \lesssim 400 \mathrm{~km} \mathrm{~s}^{-1}\right)$. The spatial distribution of $\mathrm{H} \alpha$ line widths conforms with previous observations by Swinbank et al. (2006) where the bright central source was identified as an AGN. However, since the spatial coverage of our observations does not properly extend out to the southwestern knot shown in Figure 1 (the shown contours are at the very edge of the OSIRIS FOV), we cannot confirm the possibility of two AGNs within this system, as suggested by Swinbank et al. (2006). 

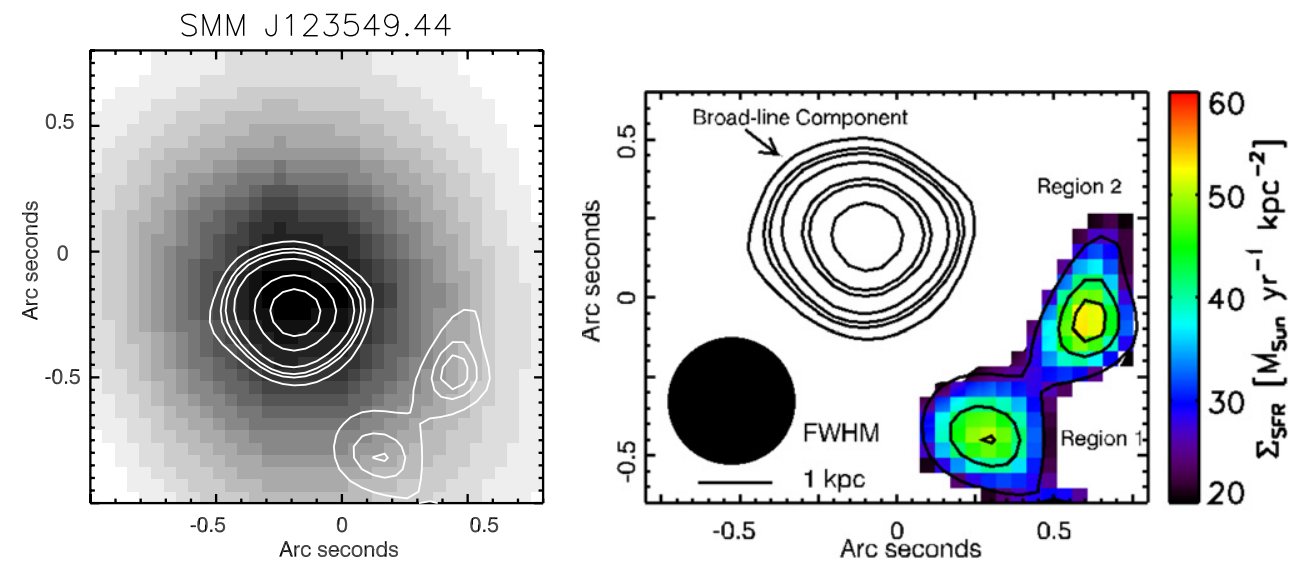

Figure 3. Distribution of $\mathrm{H} \alpha$ emission in SMM J123549.44, following the same format as Figure 1. The H $\alpha$ OSIRIS contours (inner/outer surface brightness contours corresponding to $\sim 40 / 10 \sigma$ ) are overlaid on Subaru $i$-band imaging from the Hawaii Hubble Deep Field North. The H $\alpha$ emission is dominated by a compact source, with two fainter $\mathrm{H} \alpha$ clumps roughly $\sim 0$.' 4 the southwest.

(A color version of this figure is available in the online journal.)

The velocity map in Figure 2 suggests a kinematically rich structure, where the $\mathrm{H} \alpha$ clumps to the southwest are offset from the compact component by $\sim 200-600 \mathrm{~km} \mathrm{~s}^{-1}$. These velocity offsets are distinct from the velocity found by Swinbank et al. (2006) for the secondary compact clump that they associate with a secondary AGN in the system $\left(v \sim 90 \pm 20 \mathrm{~km} \mathrm{~s}^{-1}\right)$. This suggests that the extended $\mathrm{H} \alpha$ emission is not directly associated with the secondary continuum source to the southwest of the bright point source.

The $\mathrm{H} \alpha$ arising from the OSIRIS-detected bright point source accounts for $\sim 90 \%$ of the clump-integrated $\mathrm{H} \alpha$ observed in the diffuse region. We note that this contribution from the AGN to the $\mathrm{H} \alpha$ emission is an upper limit because (1) we clearly do not cover the full extent of the SMG, (2) we are not sensitive to fainter emission, and (3) we have no constraints on the potential spatially inhomogeneous dust extinction that has been shown to be significant in SMGs (Swinbank et al. 2010).

\subsubsection{SMM J123549.44}

Ultra-deep Chandra observations of this source have revealed clear AGN signatures (Alexander et al. 2005). Furthermore, recent mid-IR spectroscopic (Menéndez-Delmestre et al. 2009) and photometric observations (Hainline et al. 2011) have revealed an excess of hot dust continuum emission, in agreement with the hypothesis of a significant contribution from AGN activity to the bolometric luminosity. Tacconi et al. $(2006,2008)$ have also undertaken high-resolution $\mathrm{CO}$ observations of this galaxy and find $\mathrm{CO}$ emission dominated by a compact source $\left(\lesssim 0^{\prime \prime} 5\right)$ with a prominent double-peaked CO profile, which they associate with the orbital motions of gas within a disk close to an AGN.

Optical and near-IR (long-slit) spectroscopy set this object at $z=2.203$ (Chapman et al. 2005; Swinbank et al. 2004). Figure 3 shows that the $\mathrm{H} \alpha$ emission for SMM J123549.44 is dominated by a compact source with $\mathrm{FWHM} \simeq 0^{\prime} .24(3 \mathrm{kpc})$, though faint traces of diffuse emission extend out to $0 ! 5(\sim 4 \mathrm{kpc})$ to the southwest of the central region. From long-slit observations, Swinbank et al. (2004) report a relatively narrow- $\mathrm{H} \alpha$ emission of $\mathrm{FWHM}_{\mathrm{rest}}=540 \pm 30 \mathrm{~km} \mathrm{~s}^{-1}$. In contrast, Harrison (2012) identify in the galaxy-wide [O III] spectrum an extremely broadline emission ( FWHM $=1500 \mathrm{~km} \mathrm{~s}^{-1}$ ) attributed to a possible AGN outflow, while a hint of extended [O III] emission $(\lesssim 0$ ' 8$)$ is reported with $\mathrm{S} / \mathrm{N}$ insufficient for proper constraints on its spectral properties.
Our observations unveil the $\mathrm{H} \alpha$ line-width distribution shown in Figure 4, allowing us to clearly distinguish broad- $\mathrm{H} \alpha$ line emission arising from a bright compact region, with FWHM = $1260 \pm 210 \mathrm{~km} \mathrm{~s}^{-1}$ and a velocity offset of $\sim-150 \pm 50 \mathrm{~km} \mathrm{~s}^{-1}$ from the redshift reported by Swinbank et al. (2004). Narrower $\mathrm{H} \alpha$ emission $\left(\mathrm{FWHM} \sim 500-800 \mathrm{~km} \mathrm{~s}^{-1}\right.$ ) characterizes the two fainter $\mathrm{H} \alpha$ clumps to the southwest (see Figure 4; Table 2). The contribution from the broad- $\mathrm{H} \alpha$ region to the total emission within the H $\alpha$-bright clumps amounts to $\sim 90 \%$, keeping in mind similar caveats stated for SMM J030227.73. Adopting the broad-line redshift as that of the system, we report a redshift of $z=2.2012$.

The velocity map shown in Figure 4 suggests internal velocity offsets of $\sim 100-200 \mathrm{~km} \mathrm{~s}^{-1}$, consistent with the velocities reported by Tacconi et al. (2008). Although these velocities could reflect the ordered motions of a gaseous disk, resulting in a total dynamical mass $M_{\text {dyn }}=\sigma^{2} R / G \sim 4 \times 10^{10} M_{\odot}$, the asymmetrical disposition of the narrow-line $\mathrm{H} \alpha$-bright clumps around the broad-line region suggests that this is not a wellbehaved disk structure with an AGN at its center. Hence, the velocity offsets could also be attributed to the relative motion of multiple galactic-scale companions, potentially undergoing a merger. However, the low $\mathrm{S} / \mathrm{N}$ of the southern and western components and the potential for an underlying lower surfacebrightness plane of emission below the instrument's sensitivities remain an important caveat.

\subsubsection{SMM J163650.43}

This galaxy was first detected by Scott et al. (2002) and Ivison et al. (2002). Its broadband near-IR emission has been studied in detail by Smail et al. (2003). With a bolometric luminosity $L_{\text {bol }}=(3 \pm 2) \times 10^{13} L_{\odot}$, this galaxy is unusually luminous even for an SMG (Chapman et al. 2003). Optical and near-IR spectroscopy revealed a redshift of $z \simeq 2.38$ (Chapman et al. 2003) and unveiled narrow-H $\alpha$ emission with an underlying broad-H $\alpha$ component $\left(\mathrm{FWHM}_{\mathrm{rest}} \simeq 310 \pm 50\right.$ and $1750 \pm 240 \mathrm{~km} \mathrm{~s}^{-1}$, respectively; Swinbank et al. 2004) and a high $[\mathrm{O}$ III $] / \mathrm{H} \beta$ line ratio characteristic of a Seyfert AGN (Smail et al. 2003). SMM J163650.43 has also been identified as an AGN-dominated SMG based on the $\left[S_{8 \mu \mathrm{m}} / S_{4.5 \mu \mathrm{m}}>2\right]$ IRAC color selection, with a $69 \%$ AGN contribution to the mid-IR emission based on mid-IR spectral decomposition (see Coppin et al. 2010). This galaxy has been resolved into different components, revealing a complex structure (Smail et al. 

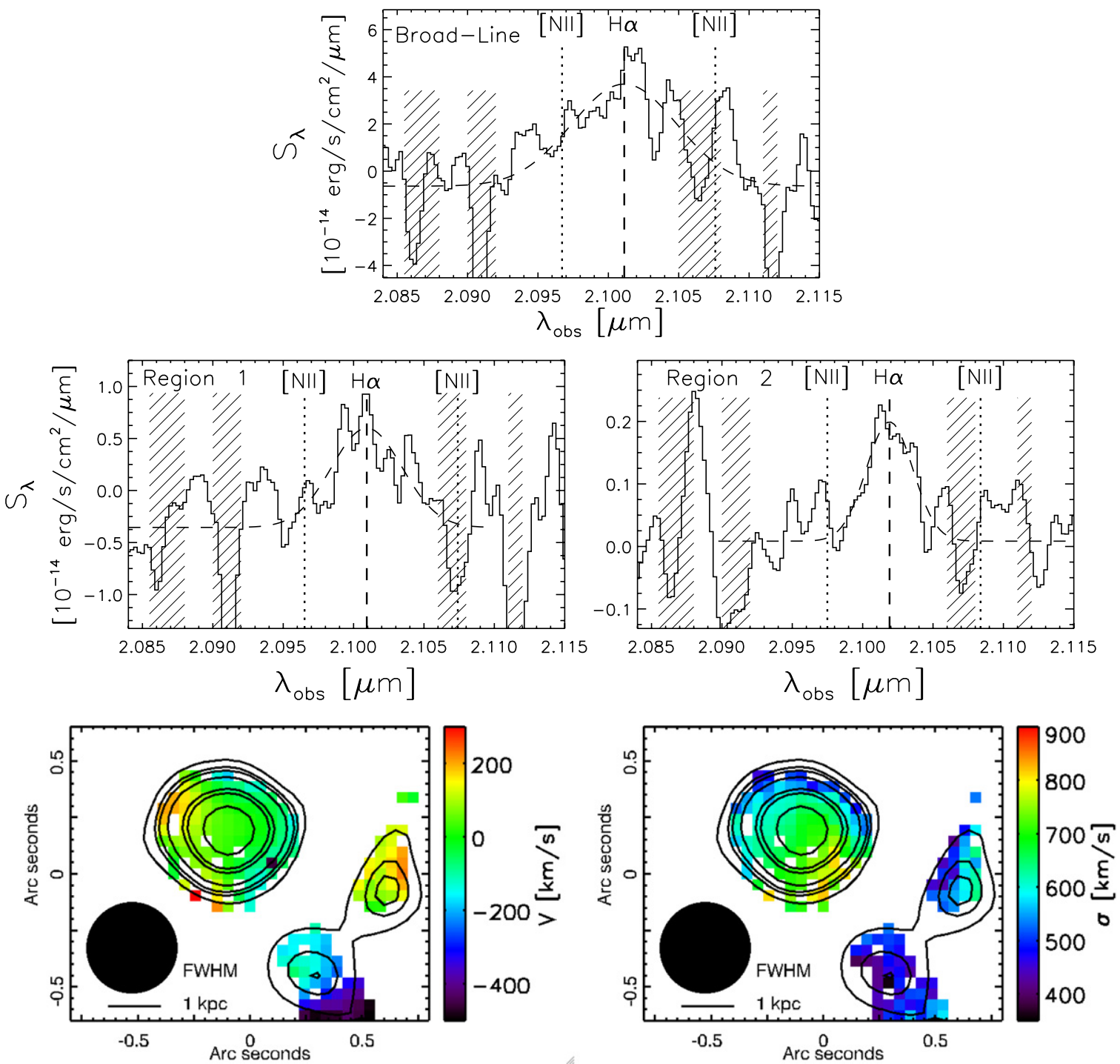

Figure 4. $\mathrm{H} \alpha$ kinematics in SMM J123549.44, following the same format as Figure 2. The bright point-source emission that dominates the H $\alpha$ intensity map displays broad-H $\alpha$ emission at a redshift $z=2.201$ and is likely associated with an AGN. At a fainter S/N, the two distinct components to the southwest (see Figure 3) show narrower $\mathrm{H} \alpha$ line emission and are likely dominated by star formation activity (see Table 2).

(A color version of this figure is available in the online journal.)

2003; Swinbank et al. 2005, 2006). In particular, based on recent seeing-limited Gemini-North NIFS observations, Harrison (2012) report intricate extensions of narrow [O III] emission $\left(100 \lesssim \mathrm{FWHM} \lesssim 500 \mathrm{~km} \mathrm{~s}^{-1}\right)$ and two kinematically distinct broad [O III] emission lines (FWHM $\sim 1200 \mathrm{~km} \mathrm{~s}^{-1}$ ). Furthermore, subarcsecond resolution $(\sim 0$ '.25-0.5) CO observations of SMM J163650.43 undertaken by Tacconi et al. (2008) show a two-peaked $\mathrm{CO}$ profile emission restricted to an elliptical region with an intrinsic FWHM size of $0 . .8 \pm 0.2 \times 0.2 \pm 0.3$.

As shown in Figure 5, we find $\mathrm{H} \alpha$ emission in the shape of an arc, extending over $\sim 5 \times 7 \mathrm{kpc}^{2}$ and tracing the continuum emission. The OSIRIS $\mathrm{H} \alpha$ morphology agrees very well with the IFU results on this galaxy by Swinbank et al. (2005), where the seeing-limited observations show three separate components corresponding to the northern, the western, and the southern portions of the arc in Figure 5. Aided with AO, the OSIRIS lenslet scale 0 .'05 allows us to zoom further into the details of the inner regions of this galaxy: we distinguish five regions indicated in Figure 5. At an $\mathrm{S} / \mathrm{N}>10$, we distinguish broad-H $\alpha$ emission associated with the northeastern component coinciding with the red knot visible in the underlying $H S T$ image (see Figures 5 and 6). With a line width of $\sigma \sim 1000-1500 \mathrm{~km} \mathrm{~s}^{-1}$ (FWHM $\gtrsim$ $3000 \mathrm{~km} \mathrm{~s}^{-1}$; see Table 2), we associate this emission to the broad-line region of an AGN. The two broad [O III] components identified by Harrison (2012) are spatially coincident with the broad-H $\alpha$ region in our OSIRIS observations, but the velocity offsets ( $\Delta v= \pm 850 \mathrm{~km} \mathrm{~s}^{-1}$ ) associated to these components suggest that the [O III] broad emission traces bi-polar outflows driven by the AGN.

We find narrower $\mathrm{H} \alpha$ emission from Region 1 and from the western and southern regions of the arc (Regions 2, 3, and 4), with $\sigma \lesssim 300 \mathrm{~km} \mathrm{~s}^{-1}$ (FWHM $<700 \mathrm{~km} \mathrm{~s}^{-1}$; see Table 2). Close to $\sim 35 \%$ of the clump-integrated $\mathrm{H} \alpha$ emission detected by OSIRIS lies within the extent of the broad-line region associated to the AGN (FWHM $\simeq 0^{\prime} .2 \sim 1.7 \mathrm{kpc}$ ), while the remainder of the $\mathrm{H} \alpha$ is spread to larger scales, with each of the $1-2 \mathrm{kpc}$ sized $\mathrm{H} \alpha$-bright clumps accounting for $\sim 3 \%-30 \%$. Based on the 1D spectra shown in Figure 6, we find velocity offsets corresponding to $\sim 100-200 \mathrm{~km} \mathrm{~s}^{-1}$ between the different regions, in good agreement with previous findings by Swinbank et al. (2005). 

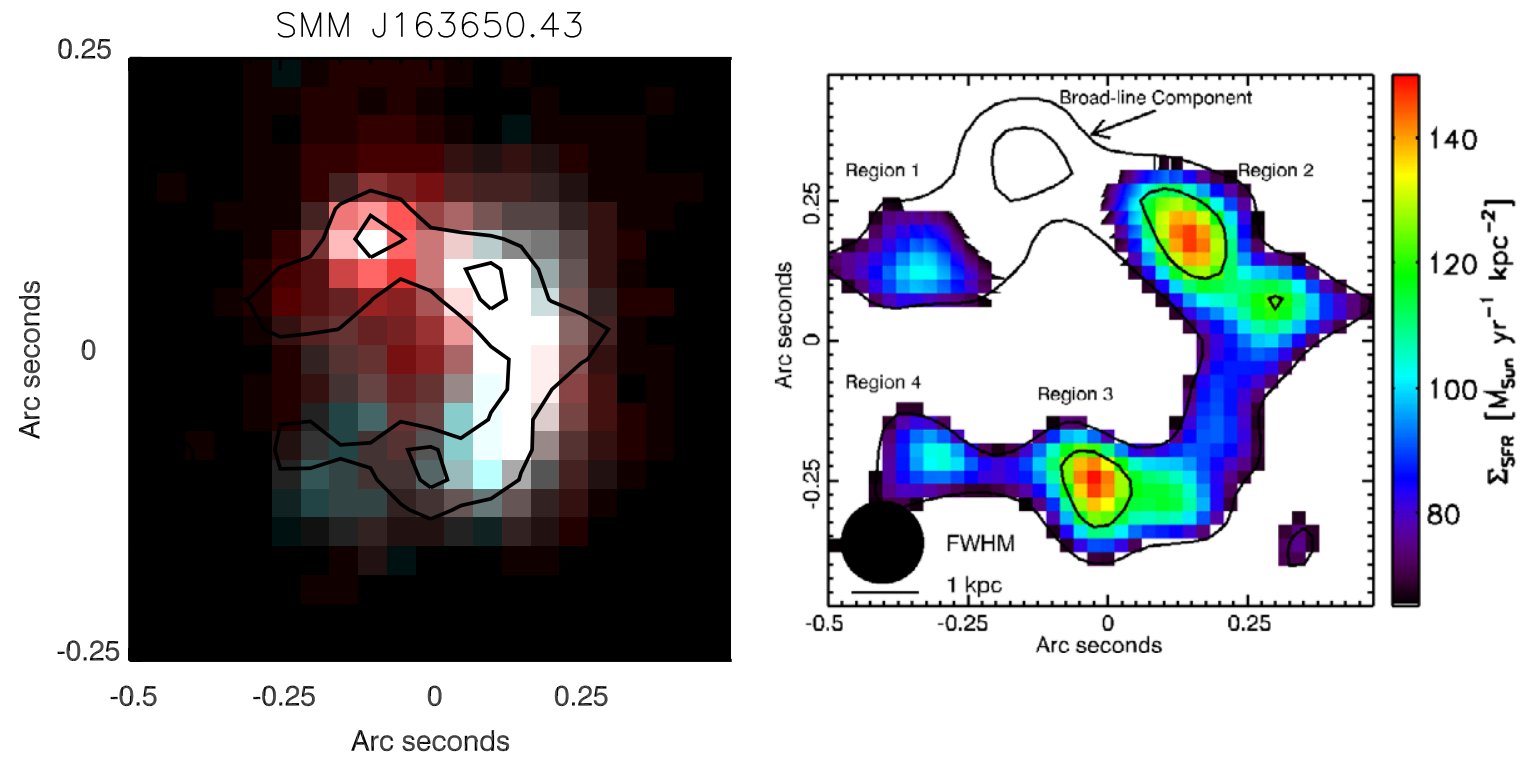

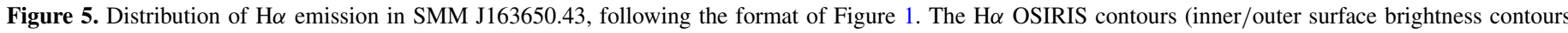

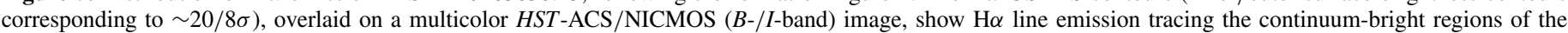

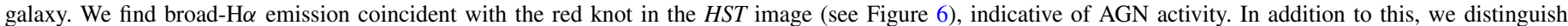
$\mathrm{H} \alpha$-bright clumps within the diffuse $\mathrm{H} \alpha$ component. We divide this component into four distinct regions and study them individually.

(A color version of this figure is available in the online journal.)

\subsection{Star Formation Rate Surface Density in SMGs}

Based on Kennicutt (1998), we derive a local SFR at each spatial pixel in our flux-calibrated $\mathrm{H} \alpha$ images for each SMG target. Taking into account the pixel scale of our observations (see Table 1 ), we construct the SFR surface density $\left(\Sigma_{\mathrm{SFR}}\right)$ maps shown in Figures 1, 3, and 5. The regions of broad-H $\alpha$ emission, which we assume to be associated with AGN activity, have been subtracted following the approach described in Section 2.1 to cleanly eliminate the AGN contribution. The remaining narrowline $\mathrm{H} \alpha$ emission shown in these figures, likely associated with star formation, extends out to scales $>0$ '. $5-2^{\prime \prime}(\sim 4-16 \mathrm{kpc})$. However, the bulk of this emission appears to be concentrated in multiple $\mathrm{H} \alpha$ unresolved clumps, limited in size by the PSF FWHM $\sim 0$.'10-0.'25 (1-2 kpc at $z \sim 2)$.

We note that although the near-IR provides us with a lessobscured insight to these SMG clumps than the optical, the presence of significant obscuration remains an important caveat (Takata et al. 2006). In the absence of spatially resolved extinction maps, we make an estimated extinction correction based on the typical Balmer decrement found for SMGs: Takata et al. (2006) have shown that SMGs typically have observed Balmer decrements $\mathrm{H} \alpha / \mathrm{H} \beta \sim$ 5-20, corresponding to extinction levels in the range $A_{v} \sim 1-4$ with a median $\left\langle A_{v}\right\rangle=2.9 \pm 0.5$. Assuming a typical Balmer decrement $\sim 10$, we apply the corresponding reddening correction of $e^{\tau_{\mathrm{Balmer}}} \sim 4$ to the measured $\mathrm{H} \alpha$ luminosities. Note that this factor is significantly larger than the typical attenuation found for other high-redshift populations, such as the optically selected LBGs, with attenuations of an average factor of 1.7 (Erb et al. 2006).

Prior to extinction correction, SMG clumps already have large SFR surface densities with peak values $\Sigma_{\text {SFR }} \sim 1-50$ (see Figures 1, 3, and 5). After correcting for extinction in the manner we described, the peak SFR surface densities increase up to $\Sigma_{\mathrm{SFR}} \sim 200 M_{\odot} \mathrm{yr}^{-1} \mathrm{kpc}^{-2}$ and the clump-wide SFRs reach values of up to $250 M_{\odot} \mathrm{yr}^{-1}$. In Figure 7 , we show the total SFRs and SFR surface densities as a function of the line velocity dispersions based on the clump-wide spectral extractions shown in Figures 2, 4, and 6, with the extinctioncorrected values shown by the endpoints of horizontal arrows. For comparison, we include both kiloparsec-scale and galaxywide measurements for optically and near-IR selected galaxies at $z \sim 2-3$ (Lehnert et al. 2009; Förster Schreiber et al. 2009; Law et al. 2009) and for samples of low-redshift normal and starburst galaxies, compiled by Dib et al. (2006). We also compare the distribution in SFR surface densities in our SMG regions with that in low-redshift samples of normal galaxies and circumnuclear starbursts from Kennicutt (1998) and luminous infrared galaxies (LIRGs; $L_{8-1000 \mu \mathrm{m}} \sim 10^{11}-10^{12} L_{\odot}$ ) from Alonso-Herrero et al. (2006).

The SMG clump-integrated SFRs extend over a large range of values $\left(\mathrm{SFR}_{\text {clump }} \sim 1-60 M_{\odot} \mathrm{yr}^{-1}\right)$ and are similar to the galaxy-wide values that have been attributed to LBGs (Law et al. 2009) and other high-redshift galaxies from the SINS sample (e.g., Förster Schreiber et al. 2009), particularly when extinction correction is taken into account (see left panel in Figure 7). It has been shown that a trend exists between $\Sigma_{\mathrm{SFR}}$ and $\mathrm{H} \alpha$ velocity dispersion in these high-redshift galaxy populations, where the most intense star-forming regions are also those with the highest velocity dispersions (Lehnert et al. 2009; Law et al. 2009; Förster Schreiber et al. 2009). On the right panel of Figure 7, we show that the SMG clumps in our sample occupy the high end of this trend. Although the SMG clumps in this sample present a wide range of extinction-corrected SFR and $\Sigma_{\mathrm{SFR}}$ values $(\mathrm{SFR} \sim$ 3-250 $M_{\odot} \mathrm{yr}^{-1}, \Sigma_{\mathrm{SFR}} \sim 1-50 M_{\odot} \mathrm{yr}^{-1} \mathrm{kpc}^{-2}$ ), the average values that characterize the star formation activity in these $\left(\langle\mathrm{SFR}\rangle \sim 60 M_{\odot} \mathrm{yr}^{-1},\left\langle\Sigma_{\mathrm{SFR}}\right\rangle \sim 10 M_{\odot} \mathrm{yr}^{-1} \mathrm{kpc}^{-2}\right)$ is similar in intensity - though higher by a factor of $2-5$ in some cases - to that found in other high-redshift populations (e.g., $\langle\mathrm{SFR}\rangle_{\mathrm{LBGs}} \sim$ $25 M_{\odot} \mathrm{yr}^{-1},\left\langle\Sigma_{\mathrm{SFR}}\right\rangle_{\mathrm{LBGs}} \sim 4 M_{\odot} \mathrm{yr}^{-1} \mathrm{kpc}^{-2}$; Law et al. 2009). However, the $\mathrm{H} \alpha$ velocity dispersions are significantly different, likely associated to the different mass ranges that these different galaxy populations correspond to. Assuming that $\sigma_{\mathrm{H} \alpha}$ traces gas dynamics within virialized clouds and considering a typical clump size of $1 \mathrm{kpc}$, we determine clump dynamical masses 
Table 2

Summary of OSIRIS IFU Results

\begin{tabular}{|c|c|c|c|}
\hline & SMM J030227.73 & SMM J123549.44 & SMM J163650.43 \\
\hline \multicolumn{4}{|c|}{$S_{\mathrm{H} \alpha}\left(10^{-17} \mathrm{erg} \mathrm{s}^{-1} \mathrm{~cm}^{-2}\right)^{\mathrm{a}}$} \\
\hline Region 1 & $0.9 \pm 0.4$ & $6.0 \pm 3.0$ & $1.7 \pm 1.2$ \\
\hline Region 2 & $2.0 \pm 0.9$ & $0.8 \pm 0.4$ & $17.0 \pm 8.0$ \\
\hline Region 3 & $0.9 \pm 0.5$ & $\cdots$ & $16.5 \pm 3.0$ \\
\hline Region 4 & $0.7 \pm 0.6$ & $\cdots$ & $3.7 \pm 2.0$ \\
\hline Broad-line region & $59.0 \pm 21.0$ & $110.0 \pm 23.0$ & $20.0 \pm 4.0$ \\
\hline \multicolumn{4}{|l|}{$\operatorname{SFR}_{\mathrm{H} \alpha}\left(M_{\odot} \mathrm{yr}^{-1}\right)^{\mathrm{b}}$} \\
\hline Region 1 & 0.9 & 17.0 & 6.0 \\
\hline Region 2 & 1.9 & 2.3 & 60.0 \\
\hline Region 3 & 0.8 & $\cdots$ & 58.0 \\
\hline Region 4 & 0.7 & $\cdots$ & 13.0 \\
\hline \multicolumn{4}{|l|}{$\Sigma_{\mathrm{SFR}}\left(M_{\odot} \mathrm{yr}^{-1} \mathrm{kpc}^{-2}\right)$} \\
\hline Region 1 & 0.4 & 6.6 & 12.0 \\
\hline Region 2 & 0.3 & 13.0 & 10.0 \\
\hline Region 3 & 0.4 & $\cdots$ & 7.0 \\
\hline Region 4 & 0.9 & $\cdots$ & 5.0 \\
\hline \multicolumn{4}{|l|}{ FWHM $_{\text {rest }}\left(\mathrm{km} \mathrm{s}^{-1}\right)$} \\
\hline Region 1 & $190 \pm 60$ & $840 \pm 340$ & $330 \pm 160$ \\
\hline Region 2 & $400 \pm 130$ & $550 \pm 190$ & $700 \pm 220$ \\
\hline Region 3 & $260 \pm 100$ & $\cdots$ & $390 \pm 50$ \\
\hline Region 4 & $370 \pm 230$ & $\cdots$ & $470 \pm 170$ \\
\hline Broad-line region & $3100 \pm 650$ & $1260 \pm 210$ & $2660 \pm 390$ \\
\hline
\end{tabular}

Notes.

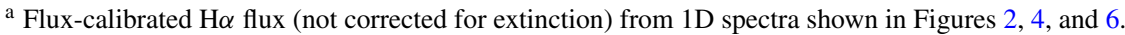

b Based on Kennicutt (1998).

in the range of $M_{\text {clumps }}=\sigma^{2} R / G \sim 1-30 \times 10^{9} M_{\odot}$. These clump masses are $\sim 1-2$ orders of magnitude larger than the kiloparsec-scaled stellar clumps uncovered by Förster Schreiber et al. (2011) in the optically selected sample of $z \sim 2$ SINS starforming galaxies $\left(M_{\text {clumps }} \sim 0.1-8 \times 10^{9} M_{\odot}\right)$.

Lehnert et al. (2009) argue that the relationship between SFR surface density and the ionized gas velocity dispersion is a consequence of star formation self-regulation, where the mechanical energy of the starburst itself controls the local dynamics of the ionized gas. Following Lehnert et al. (2009), this relationship can be represented by a functional form involving the intensity of star formation with the velocities of the ionized gas to represent the energy injected into the interstellar medium surrounding the star-forming regions (see curve in Figure 7). Within this context, the high velocity dispersions observed in our SMG clumps are likely not the sole result of larger dynamical masses. They are likely a combination of differences in dynamical mass ranges and a reflection of the high pressures sustained by high local surface brightnesses, which in turn are likely due to the higher gas densities in SMGs (e.g., Harris et al. 2010).

Compared to galaxies at low redshift, the right panel of Figure 7 shows that SMG clumps have $\Sigma_{\text {SFR }}$ values $>3$ orders of magnitude higher than those found in normal spirals (Kennicutt 1998; Dib et al. 2006), but which fall well within the range of starburst galaxies (Kennicutt 1998). If we correct for extinction the $\Sigma_{\mathrm{SFR}}$ in SMG clumps are shifted at the most within a factor of a few from the range occupied by the typical local circumnuclear starbursts, similar to the range found in low-redshift LIRGs (Alonso-Herrero et al. 2006).

\section{DISCUSSION}

Near-IR integral-field spectroscopic observations have shown that $\mathrm{H} \alpha$ emission in SMGs often hint to the presence of multiple spatially distinct galactic-scale subcomponents within the central $\sim 1^{\prime \prime}$ (Swinbank et al. 2006; Nesvadba et al. 2007). With LGS-AO OSIRIS, we have been able to peer into these central regions, allowing us to zoom in further than these previous seeing-limited integral-field observations.

\subsection{Internal Dynamics: SMGs are Kinematically Disturbed Systems}

In all three SMGs of our sample, we identify regions with distinct properties: regions with narrow- $\mathrm{H} \alpha$ emission where star formation is likely taking place and regions with broad$\mathrm{H} \alpha$ emission, likely dominated by AGN activity (keeping in mind that outflows may also play a role; e.g., Shapiro et al. 2008, 2009; Newman et al. 2012). We find that the narrow$\mathrm{H} \alpha$ emission is concentrated in multiple clumps unresolved on $\sim 1-2 \mathrm{kpc}$ scales, asymmetrically distributed around the AGN. These clumps suggest either one of the following scenarios: (1) these $\mathrm{H} \alpha$-bright regions correspond to distinct components in a disturbed system, as would be found in a merging system; or, (2) we are picking out the high surface-brightness peaks of $\mathrm{H} \alpha$ emission in an underlying lower surface-brightness plane of emission-perhaps even a disk, as has been found in a subsample of massive optically selected star-forming galaxies at $z \sim 2-3$ (Förster Schreiber et al. 2006, 2011; Law et al. 2007b, 2009).

We find velocity offsets of $\sim$ few $\times 100 \mathrm{~km} \mathrm{~s}^{-1}$ between distinct galactic-scale regions (see Figures 2, 4, and 6). These velocity offsets could be explained by invoking a merger scenario (see also Engel et al. 2010), thus strengthening the conclusion derived from deep rest-frame optical HST-imaging that SMGs are disturbed systems, likely corresponding to mergers (Smail et al. 1998, 2004; Ivison et al. 2010; Swinbank et al. 2010). Within this context, the distinct components revealed by OSIRIS could potentially be associated with the 

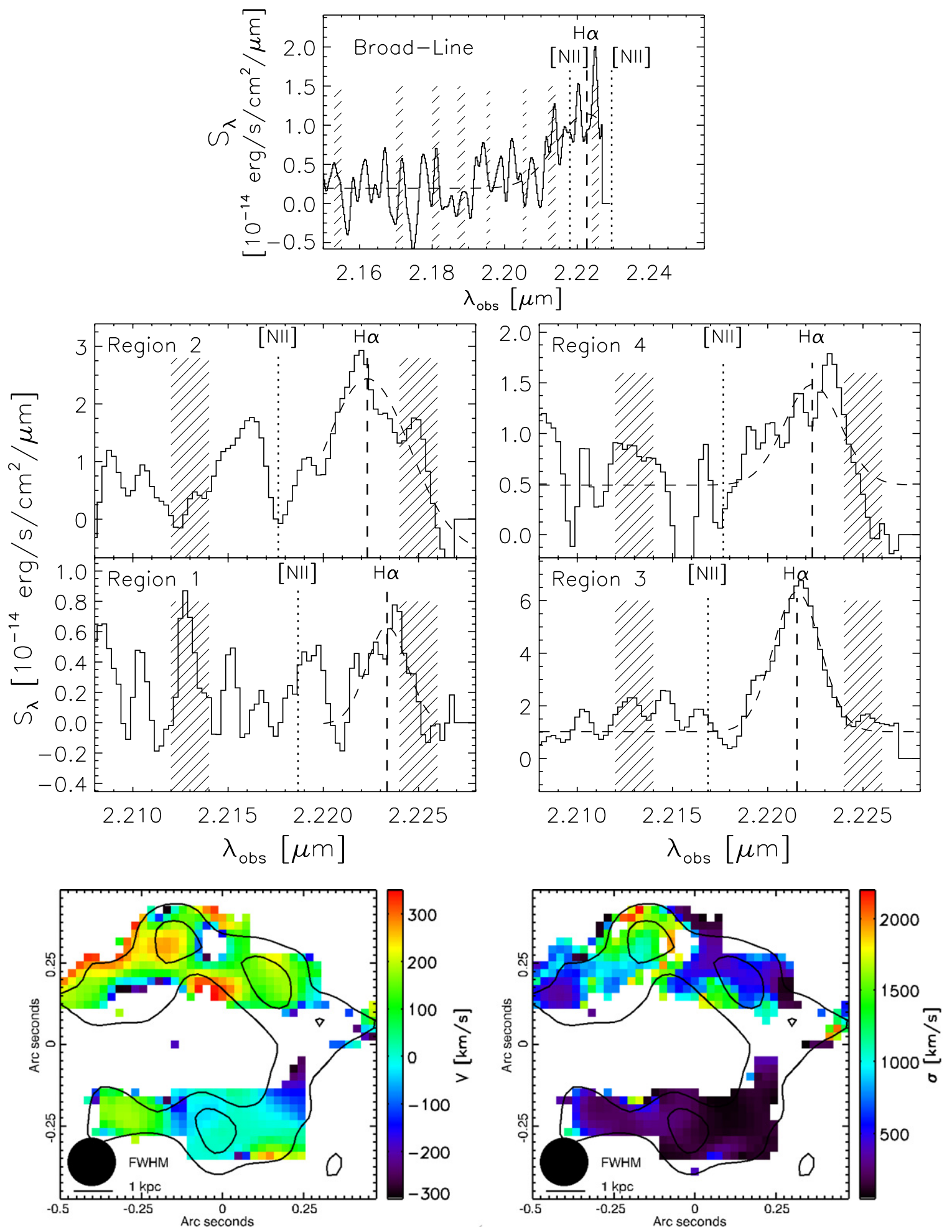

Figure 6. H $\alpha$ kinematics in SMM J163650.43, following the format of Figure 2. The 1D spectra suggest the presence of a broad-H $\alpha$ line coincident with the region that appears as a red knot of continuum emission in Figure 5; this is indicative of AGN activity.

(A color version of this figure is available in the online journal.)

remnants of the pre-merger galaxies that have not fully coalesced and still retain significant intrinsic velocities with respect to each other. This is the kinematic structure expected for the intermediate stages of a merging system (e.g., Cox et al. 2006; Hayward et al. 2011). We note that this is in agreement with Greve et al. (2005), where they discuss the likelihood that the double-peaked CO profiles observed in a sample of 18 SMGs correspond to either a disk or a merger. Taking into account estimates of gas masses and source sizes, they conclude that such high surface mass density would imply dynamical instabilities too powerful for a disk to survive. They thus conclude that the double-peaked nature of the $\mathrm{CO}$ lines traces distinct gas-rich 

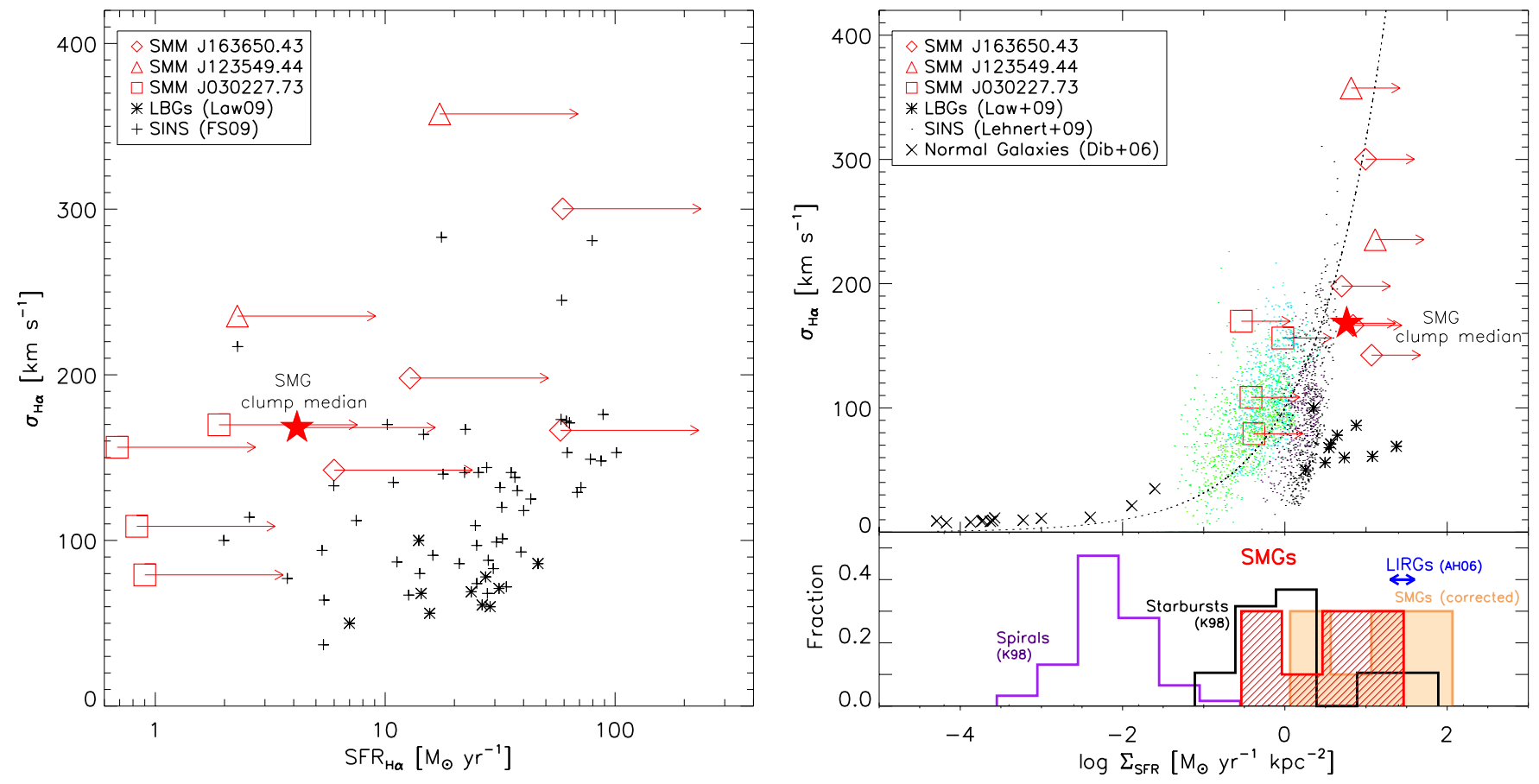

Figure 7. SFR (left panel) and SFR surface density (right panel) as a function of velocity dispersions based on $\mathrm{H} \alpha$ OSIRIS observations for individual SMG H $\alpha$-bright clumps (large symbols). The large filled star corresponds to the median value for the SMG clumps and the endpoints of the horizontal arrows indicate the result after correcting for extinction (see Section 3.2 for details). For comparison, we include values (not corrected for extinction) based on H $\alpha$ for local normal galaxies (Dib et al. 2006) and at the high-redshift end, based on galaxy-integrated $\mathrm{H} \alpha$ measurements for optically and near-IR selected galaxies at $z \sim 2-3$, including LBGs (Law et al. 2009) and galaxies from the SINS sample (Förster Schreiber et al. 2009, FS09), and on spatially resolved H $\alpha$ measurements on select SINS galaxies (Lehnert et al. 2009). The dashed curve on the right panel represents the functional form $\left.\sigma \sim \sqrt{(} \epsilon \Sigma_{\mathrm{SFR}}\right)$ presented by Lehnert et al. (2009) to represent the coupling between the injection of mechanical energy by intense star-forming activity and the kinematics of the ionized gas, with $\epsilon=240$ representing $25 \%$ coupling efficiency. To discuss SMG SFR intensities within a wider range of environments found in the low-redshift universe, we also include the distribution in SFR surface densities based on IR measurements for normal spirals and starbursts (Kennicutt 1998, K08) and those for LIRGs based on Paschen- $\alpha$ measurements in comparably extended star-forming regions ( 0.7-2 kpc; Alonso-Herrero et al. 2006, AH06). These figures show that SMGs clumps harbor very intense star formation activity, occupying the high end of the $\Sigma_{\text {SFR }}$ vs. $\sigma$ relation and sharing SFR intensities with that found in local extreme environments, such as nuclear starbursts and LIRGs.

(A color version of this figure is available in the online journal.)

components undergoing a merger or arise from a disk collapsing under gravitational instability.

On the other hand, the H $\alpha$-bright clumps of emission that OSIRIS picks out may correspond to distinct clumps within an underlying clumpy disk structure. This would be similar to the clumpy disks identified in face-on galaxies at somewhat lower redshifts $(z \sim 0.5-2.0$; Elmegreen et al. 2004a, 2004b) and the more recently identified stellar clumps in optically and nearIR selected star-forming galaxies at $z \sim 2$ by Shapiro et al. (2009), Förster Schreiber et al. (2011), Genzel et al. (2011), and Newman et al. (2012). Recent studies have indeed suggested the presence of disk structures in a large fraction of SMGs based on deep broadband imaging $K$-band data (e.g., Targett et al. 2011). Although these studies are unable to directly trace the kinematic structure of these objects, they may point to the possibility that SMGs may correspond to a diverse population presenting a varied range of kinematic structures.

Considering that the broad-H $\alpha$ emission in our SMGs identifies a supermassive black hole likely at the dynamical center of system, and the large masses and extreme star-forming properties of the SMG clumps that are in turn asymmetrically distributed around the AGN, it is unlikely that these all reside in a regular potential well structure. The merger interpretation is likely the most accurate scenario for the SMGs in our sample, although the final test of whether an underlying disk structure is present will come from studies of the cold gas at the high spatial resolutions with ALMA.

\subsection{Extended Starbursts at High Redshift}

We find compact unresolved regions (FWHM 1-3 kpc) of broad-line $\mathrm{H} \alpha$ emission that contribute a significant fraction ( $\sim 30 \%-90 \%)$ to the galaxy-wide $\mathrm{H} \alpha$ emission. However, fainter narrow-line $\mathrm{H} \alpha$ emission extends over large spatial scales of 4-16 kpc (see Figures 1, 3, and 5), with 1-2 kpc sized $\mathrm{H} \alpha$-bright clumps indicating regions of particularly intense star formation (see line-width maps in Figures 2, 4, and 6). Spatially extended sizes for the diffuse $\mathrm{H} \alpha$ emission have also been identified in the seeing-limited IFU observations presented by Swinbank et al. (2006) and Alaghband-Zadeh et al. (2012), who report resolved nebular emission on scales from 4 to $11 \mathrm{kpc}$ for a combined sample of 12 SMGs.

Evidence of spatially extended emission in SMGs has also been found at longer wavelengths. In high-resolution observations of a range of CO transitions (Engel et al. 2010; Ivison et al. 2011) and radio continuum (Biggs \& Ivison 2008), linear sizes in the range of $\sim 1-16 \mathrm{kpc}$ have been identified based on median angular-averaged FWHM sizes, with sample median sizes of 5-7 kpc. High-resolution radio continuum observations by Chapman et al. (2004) also unveil spatial extensions with a linear diameter out to $\gtrsim 10 \mathrm{kpc}$, while a high-resolution far-IR study by Younger et al. (2010) reveal far-IR emission extending out to spatial scales in the range of $\sim 5-8 \mathrm{kpc}$.

The similarity in IR luminosities between local ultraluminous infrared galaxies (ULIRGs; $L_{8}-1000 \mu \mathrm{m}>10^{12} L_{\odot}$ ) and 
high-redshift SMGs, as well as the presence of AGN signatures in both populations have naturally motivated direct comparisons and discussions of their possible correspondence within the formation and evolution scenario of today's most massive spheroids. To a somewhat still controversial degree, the sizes of SMGs also play a role in this debate. High-resolution studies of the CO and nebular emission in SMGs have concluded that SMGs have compact sizes (Tacconi et al. 2006, 2008), although often with companions with an average separation of $\sim 8 \pm 2$ (Alaghband-Zadeh et al. 2012), while other studies have demonstrated the presence of more extended spatial extensions (Chapman et al. 2004; Tecza et al. 2004; Nesvadba et al. 2007; Biggs \& Ivison 2008; Hailey-Dunsheath et al. 2010). The former argues for a similarity with local ULIRGs, which are found to be compact with sizes $\sim 1 \mathrm{kpc}$ (in the mid-IR, Charmandaris et al. 2002; Díaz-Santos et al. 2010b; and in CO, Downes \& Solomon 1998; Bryant \& Scoville 1999); the latter argues for a sharp difference with local ULIRGs. However, an important role in this controversy has been played by the tenuous definition of what is compact with respect to what is extended, particularly in the case of a clumpy system, as we find in SMGs.

In light of our OSIRIS observations, together with these previous findings, it is clear that the SMG population displays a range in spatial extensions that are often significantly larger than those of local ULIRGs. On the other hand, SMGs share similar star formation intensities with LIRGs (see Figure 7), which are still within the range of extremely active environments, yet within a somewhat lower IR luminosity bin than ULIRGs. LIRGs have also been found to display rest-frame optical line emission over large spatial scales $(\sim 3-7 \mathrm{kpc}$; AlonsoHerrero et al. 2006) as well as mid-IR extra-nuclear emission (Díaz-Santos et al. 2010a, 2010b), both indicative of extended star formation. We emphasize that evidence for extended star formation at high redshifts is not limited to SMGs. Some of the brightest and most massive LBGs do appear to have comparable $\mathrm{H} \alpha$ spatial extensions $\left(R_{\mathrm{H} \alpha} \sim 2-7 \mathrm{kpc}\right.$; Förster Schreiber et al. 2006).

Our results indicate that the SMG clumps in our sample have high surface densities of star formation activity, close to those found in local extreme environments, such as in circumnuclear starbursts and LIRGs. However, considering the much greater spatial extents found for these SMGs $(\sim 8-16 \mathrm{kpc})$ in comparison to the $1 \mathrm{kpc}$ sized nuclear starbursts (Kennicutt 1998), SMGs appear to be undergoing this intense activity on much larger spatial scales.

\section{CONCLUSIONS}

The advent of IFUs in large-aperture optical telescopes has pushed detailed galaxy kinematic studies out to the high-redshift realm. We present the first integral-field spectroscopic LGS-AO observations of SMGs. The OSIRIS instrument on Keck with LGS-AO allows us to separate spatial and spectral information at sufficiently high spatial resolutions to determine SFRs from $\mathrm{H} \alpha$-bright regions uncontaminated by the broad-line emission associated to AGN activity and to explore the internal dynamics of these complex systems. Our main results are the following:

1. We spatially distinguish between the compact broad-H $\alpha$ emission (FWHM $\sim 0$.'2-0.'4, corresponding to $\sim 2-3 \mathrm{kpc}$ ) associated with an AGN and the multiple 1-2 kpc sized narrow-H $\alpha$ clumps of emission associated with star formation, asymmetrically distributed around the AGN. This had remained unachievable in prior long-slit spectroscopic studies and seeing-limited IFU observations of SMGs.

2. We find that the $\mathrm{H} \alpha$ emission arising from the broad-line component may sometimes contribute up to $\sim 90 \%$ to the total $\mathrm{H} \alpha$ emission enclosed in the bright SMG clumps, while the contribution from the individual stellar clumps varies from $1 \%$ to $30 \%$, with a median value of $\sim 3 \%$. These contributions do not translate directly to an AGN and star formation contribution to the total luminous output, since inhomogeneous dust extinction remains unconstrained at this stage.

3. We do not find any indication of ordered global motion within our targets, as would be found in rotationally supported disks. We find relative velocities of a few $x$ $100 \mathrm{~km} \mathrm{~s}^{-1}$ between the stellar clumps and the AGN in our systems, suggesting that these SMGs do not represent regular potential well structures, but are more likely in an intermediate merging phase. However, the final test of whether an underlying disk structure is present will come from detailed studies of the cold molecular gas at the high spatial resolutions possible with ALMA.

4. SMGs seem to display high SFR surface densities $\left(\Sigma_{\mathrm{SFR}}\right)$ similar to those found in the most extreme local environments, such as circumnuclear starbursts and IR-luminous galaxies. However, because the narrow-line $\mathrm{H} \alpha$ emission spreads over large spatial extensions $\sim 4-16 \mathrm{kpc}$, this sets them in sharp contrast to local ULIRGs. All of these results taken together suggest that the submillimeter phase denotes a short-lived flaring-up of large spatial extension across these systems that rapidly depletes the available gas through intense star formation.

We thank the referee for useful comments and suggestions. We also thank David R. Law and Shelley Wright for helpful and insightful discussions on the treatment and analysis of OSIRIS observations. We are also grateful to the Keck support team for the fantastic on-site help in obtaining these observations, in particular to Al Conrad, Randy Campbell, Hien Tran, David LeMignant, Jim Lyke, and Christine Melcher. The data presented herein were obtained at the W. M. Keck Observatory, which is operated as a scientific partnership among the California Institute of Technology, the University of California and the National Aeronautics and Space Administration. The Observatory was made possible by the generous financial support of the W. M. Keck Foundation. The authors wish to recognize and acknowledge the very significant cultural role and reverence that the summit of Mauna Kea has always had within the indigenous Hawaiian community. We are most fortunate to have the opportunity to conduct observations from this mountain. K.M.D. was supported by an NSF Astronomy and Astrophysics Postdoctoral Fellowship under award AST-0802399. A.W.B. was supported by the NSF under award AST-0909159. I.R.S. acknowledges support from the Royal Society. This research has made use of the NASA/IPAC Infrared Science Archive, which is operated by the Jet Propulsion Laboratory, California Institute of Technology, under contract with the National Aeronautics and Space Administration.

\section{REFERENCES}

Alaghband-Zadeh, S., Chapman, S. C., Swinbank, A. M., et al. 2012, MNRAS, 424, 2232

Alexander, D. M., Bauer, F. E., Chapman, S., et al. 2005, ApJ, 632, 736 (A05)

Alexander, D. M., Brandt, W. N., Smail, I., et al. 2008, AJ, 135, 1968

Alonso-Herrero, A., Rieke, G. H., Rieke, M. J., et al. 2006, ApJ, 650, 835 
Barger, A. J., Cowie, L. L., Sanders, D. B., et al. 1998, Natur, 394, 248 Barger, A. J., Cowie, L. L., \& Sanders, D. B. 1999, ApJL, 518, L5 Basu-Zych, A. R., Gonçalves, T. S., Overzier, R., et al. 2009, ApJL, 699, L118 Bertoldi, F., Carilli, C. L., Menten, K. M., et al. 2000, A\&A, 360, 92 Biggs, A. D., \& Ivison, R. J. 2008, MNRAS, 385, 893

Biggs, A. D., Younger, J. D., \& Ivison, R. J. 2010, MNRAS, 408, 342 Blain, A., Smail, I., Ivison, R., Kneib, J.-P., \& Frayer, D. T. 2002, Phys. Rep., 369, 111B

Borys, C., Chapman, S., Halpern, M., \& Scott, D. 2003, MNRAS, 344, 385

Bournaud, F., Chapon, D., Teyssier, R., et al. 2011, ApJ, 730, 4

Bournaud, F., Elmegreen, B. G., \& Martig, M. 2009, ApJL, 707, L1

Bryant, P. M., \& Scoville, N. Z. 1999, AJ, 117, 2632

Chapman, S., Blain, A., Ivison, R., \& Smail, I. 2003, Natur, 422, 695

Chapman, S., Blain, A., Smail, I., \& Ivison, R. 2005, ApJ, 622, 772 (C05)

Chapman, S. C., Smail, I., Windhorst, R., Muxlow, T., \& Ivison, R. J. 2004, ApJ, 611,732

Charmandaris, V., Laurent, O., Le Floc'h, E., et al. 2002, A\&A, 391, 429

Conselice, C. J., Grogin, N. A., Jogee, S., et al. 2004, ApJL, 600, L139

Coppin, K., Halpern, M., Scott, D., Borys, C., \& Chapman, S. 2005, MNRAS, 357, 1022

Coppin, K., Pope, A., Menéndez-Delmestre, K., et al. 2010, ApJ, 713, 503

Cowie, L. L., Barger, A. J., \& Kneib, J.-P. 2002, AJ, 123, 2197

Cox, T. J., Dutta, S. N., Di Matteo, T., et al. 2006, ApJ, 650, 791

Davies, R. I. 2007, MNRAS, 375, 1099

Dekel, A., Sari, R., \& Ceverino, D. 2009, ApJ, 703, 785

Díaz-Santos, T., Alonso-Herrero, A., Colina, L., et al. 2010a, ApJ, 711, 328

Díaz-Santos, T., Charmandaris, V., Armus, L., et al. 2010b, ApJ, 723, 993

Dib, S., Bell, E., \& Burkert, A. 2006, ApJ, 638, 797

Dickinson, M., Papovich, C., Ferguson, H. C., \& Budavári, T. 2003, ApJ, 587,25

Downes, D., \& Solomon, P. M. 1998, ApJ, 507, 615

Eales, S., Lilly, S., Gear, W., et al. 1999, ApJ, 515, 518

Elmegreen, B. G., \& Elmegreen, D. M. 2005, ApJ, 627, 632

Elmegreen, D. M., Elmegreen, B. G., \& Hirst, A. C. 2004a, ApJL, 604, L21

Elmegreen, D. M., Elmegreen, B. G., \& Sheets, C. M. 2004b, ApJ, 603, 74

Engel, H., Tacconi, L. J., Davies, R. I., et al. 2010, ApJ, 724, 233

Erb, D. K., Shapley, A. E., Steidel, C. C., et al. 2003, ApJ, 591, 101

Erb, D. K., Steidel, C. C., Shapley, A. E., et al. 2006, ApJ, 647, 128

Förster Schreiber, N. M., Genzel, R., Bouché, N., et al. 2009, ApJ, 706, 1364

Förster Schreiber, N. M., Genzel, R., Lehnert, M. D., et al. 2006, ApJ, 645, 1062

Förster Schreiber, N. M., Shapley, A. E., Genzel, R., et al. 2011, ApJ, 739, 24

Genzel, R., Baker, A. J., Tacconi, L. J., et al. 2003, ApJ, 584, 633

Genzel, R., Newman, S., Jones, T., et al. 2011, ApJ, 733, 101

Gonçalves, T. S., Basu-Zych, A., Overzier, R., et al. 2010, ApJ, 724, 1373

Greve, T. R., Bertoldi, F., Smail, I., et al. 2005, MNRAS, 359, 1165

Hailey-Dunsheath, S., Nikola, T., Stacey, G. J., et al. 2010, ApJL, 714, L162

Hainline, L. J., Blain, A. W., Smail, I., et al. 2009, ApJ, 699, 1610

Hainline, L. J., Blain, A. W., Smail, I., et al. 2011, ApJ, 740, 96

Harris, A. I., Baker, A. J., Zonak, S. G., et al. 2010, ApJ, 723, 1139

Harrison, C. M. 2012, MNRAS, 426, 1073
Hayward, C. C., Kereš, D., Jonsson, P., et al. 2011, ApJ, 743, 159

Hughes, D. H., Serjeant, S., Dunlop, J., et al. 1998, Natur, 394, 241

Immeli, A., Samland, M., Gerhard, O., \& Westera, P. 2004, A\&A, 413, 547

Ivison, R. J., Greve, T. R., Smail, I., et al. 2002, MNRAS, 337, 1

Ivison, R. J., Papadopoulos, P. P., Smail, I., et al. 2011, MNRAS, 412, 1913

Ivison, R. J., Smail, I., Papadopoulos, P. P., et al. 2010, MNRAS, 404, 198

Jones, T. A., Swinbank, A. M., Ellis, R. S., Richard, J., \& Stark, D. P. 2010, MNRAS, 404, 1247

Kennicutt, R. C., Jr. 1998, ApJ, 498, 541

Laird, E. S., Nandra, K., Pope, A., \& Scott, D. 2010, MNRAS, 401, 2763

Larkin, J., \& Versteegh, A. 2006, Proc. SPIE, 6269, 62691A

Law, D. R., Steidel, C. C., Erb, D. K., et al. 2007a, ApJ, 656, 1

Law, D. R., Steidel, C. C., Erb, D. K., et al. 2007b, ApJ, 669, 929

Law, D. R., Steidel, C. C., Erb, D. K., et al. 2009, ApJ, 697, 2057

Lehnert, M. D., Nesvadba, N. P. H., Tiran, L. L., et al. 2009, ApJ, 699, 1660

Lilly, S. J., Eales, S. A., Gear, W. K. P., et al. 1999, ApJ, 518, 641

Melbourne, J., Bussman, R. S., Brand, K., et al. 2009, AJ, 137, 4854

Menéndez-Delmestre, K., Blain, A. W., Smail, I., et al. 2009, ApJ, 699, 677

Nesvadba, N. P. H., Lehnert, M. D., Genzel, R., et al. 2007, ApJ, 657, 725

Newman, S., Shapiro Griffin, K., Genzel, R., et al. 2012, ApJ, 752, 111

Overzier, R. A., Heckman, T. M., Schiminovich, D., et al. 2010, ApJ, 710, 979

Pérez-González, P. G., Rieke, G. H., Villar, V., et al. 2008, ApJ, 675, 234

Pope, A., Chary, R.-R., Alexander, D. M., et al. 2008, ApJ, 675, 1171

Scott, S., Fox, M. J., Dunlop, J. S., et al. 2002, MNRAS, 331, 817

Shapiro, K., Genzel, R., Förster, S., et al. 2008, ApJ, 682, 231

Shapiro, K., Genzel, R., Quataert, E., et al. 2009, ApJ, 701, 955

Smail, I., Chapman, S. C., Blain, A. W., \& Ivison, R. J. 2004, ApJ, 616, 71

Smail, I., Chapman, S. C., Ivison, R. J., et al. 2003, MNRAS, 342, 1185

Smail, I., Ivison, R., \& Blain, A. 1997, ApJ, 490L, 5S

Smail, I., Ivison, R. J., Blain, A. W., \& Kneib, J.-P. 1998, ApJL, 507, L21

Stark, D. P., Swinbank, A. M., Ellis, R. S., et al. 2008, Natur, 455, 775

Swinbank, A. M., Chapman, S. C., Smail, I., et al. 2006, MNRAS, 371, 465

Swinbank, A. M., Smail, I., Bower, R. G., et al. 2005, MNRAS, 359, 401

Swinbank, A. M., Smail, I., Chapman, S., et al. 2004, ApJ, 617, 64

Swinbank, A. M., Smail, I., Chapman, S. C., et al. 2010, MNRAS, 405, 234

Tacconi, L. J., Genzel, R., Smail, I., et al. 2008, ApJ, 680, 246

Tacconi, L. J., Neri, R., Chapman, S. C., et al. 2006, ApJ, 640, 228

Takata, T., Sekiguchi, K., Smail, I., et al. 2006, ApJ, 651, 713

Targett, T. A., Dunlop, J. S., McLure, R. J., et al. 2011, MNRAS, 412, 295

Tecza, M., Baker, A. J., Davies, R. I., et al. 2004, ApJL, 605, L109

van Dam, M. A., Bouchez, A. H., Le Mignant, D., et al. 2006, PASP, 118, 310

Wardlow, A., Smail, I., Coppin, K. E. K., et al. 2011, MNRAS, 415, 1479

Webb, T. M., Eales, S. A., Lilly, S. J., et al. 2003a, ApJ, 587, 41

Webb, T. M. A., Lilly, S., Clements, D. L., et al. 2003b, ApJ, 597, 680

Weiß, A., Kovács, A., Coppin, K., et al. 2009, ApJ, 707, 1201

Wisnioski, E., Glazebrook, K., Blake, C., et al. 2012, MNRAS, 422, 3339

Wizinowich, P. L., Le Mignant, D., Bouchez, A. H., et al. 2006, PASP, 118, 297

Wright, S. A., Larkin, J. E., Law, D. R., et al. 2009, ApJ, 699, 421

Younger, J. D., Fazio, G. G., Ashby, M. L. N., et al. 2010, MNRAS, 407, 1268

Younger, J. D., Fazio, G. G., Huang, J.-S., et al. 2007, ApJ, 671, 1531 